Société d'histoire de la révolution de 1848 et des

révolutions du XIXe siècle

$22 \mid 2001$

Autour de Décembre 1851

\title{
Colloque : Comment meurt une République? Autour du Deux-Décembre. Programme
}

Colloque de la Société d'histoire de la révolution de 1848, Lyon, 28, 29, 30 novembre et 1 er décembre 2001

\section{(2) OpenEdition}

Journals

Édition électronique

URL : http://journals.openedition.org/rh19/285

DOI : $10.4000 /$ rh 19.285

ISSN : $1777-5329$

Éditeur

La Société de 1848

Édition imprimée

Date de publication : 1 juin 2001

ISSN : 1265-1354

Référence électronique

"Colloque : Comment meurt une République? Autour du Deux-Décembre. Programme », Revue d'histoire du XIXe siècle [En ligne], 22 | 2001, mis en ligne le 04 septembre 2008, consulté le 15 septembre 2020. URL : http://journals.openedition.org/rh19/285

Ce document a été généré automatiquement le 15 septembre 2020.

Tous droits réservés 


\section{Colloque : Comment meurt une République? Autour du Deux- Décembre. Programme}

Colloque de la Société d'histoire de la révolution de 1848, Lyon, 28, 29, 30 novembre et 1er décembre 2001

\section{PROGRAMME}

Mercredi 28 novembre

$9 \mathrm{~h} 45$

Accueil et introduction générale du colloque : Jean-Luc MAYAUD

$10 h 05$

Thème 1 : ÉCRITURE DU COUP D'ÉTAT ET CONSTRUCTION DE L'ÉVÉNEMENT

Présidence et introductions : Michèle RIOT-SARCEY

Conclusions : Sophie WAHNICH

- Construction sociale et historique de l'événement

Jean-Claude CARON

Face au coup d'État : construction et historicisation du Deux décembre

Christophe VOILLIOT

Écriture préfectorale et construction sociale de l'événement à propos de l'"insurrection" de Clamecy

Groupe d'analyse politique, Bernard LACROIX

Du coup de force au coup d'État. Contribution à l'étude du travail de mise en forme des événements du 2 décembre 1851

$11 \mathrm{~h} 20$

Conclusion - discussions

$14 h 00$

- L'écriture et le coup d'état, entre exil et histoire

Sylvie APRILE 
Faire vivre la République. Paroles et écrits des exilés au lendemain du coup d'État Pierre SERNA

"Dix siècles passés à genoux ne se corrigent pas en trois ans". Quinet devant les coups d'État des Napoléon

Nathalie RICHARD

Coup d'état politique et révisions historiographiques: les conséquences du Deux décembre sur l'écriture de l'histoire

$15 h 10$

Conclusion - discussions

$16 h 10$

- Enjeux et constructions de la mémoire

Alain GARRIGOU

Mourir pour des idées. Les luttes d'interprétation de la mort d'Alphonse Baudin (1852-1868)

Nathalie BAYON

L'héritage tronqué. La Seconde république et le coup d'État sous la plume d'un républicain opportuniste, Eugène Spuller

17 h00

Conclusion - discussions

\section{Jeudi 29 novembre}

$8 \mathrm{~h} 45$

Thème 2 : NÉCESSITÉ, INTÉRÊT ET LÉGITIMATION DU COUP D'ÉTAT

Présidence et introductions : Sylvie APRILE

Conclusions : Sandrine KOTT

\section{- Production politique et sociale de la nécessité}

Jean-Yves MOLLIER

Le crime du 2 décembre, un événement annoncé, prédit, dénoncé et raconté avant qu'il n'ait eu lieu, ou le refus obstiné d'entendre les Cassandre

Anne GIROLLET

L'échec des dispositions constitutionnelles adoptées afin d'éviter un retour au césarisme

Jean-Claude BUSSIÈRE

Le coup d'État comme couronnement de l'élection

10 hoo

Conclusion - discussions

11 h0o

\section{- Production idéologique de l'intérêt}

Alice PRIMI

Le coup d'État : un espoir de progrès social et de régénération chrétienne

François FOURN

1849-1851 : l'anticommunisme en France. Le spectre rouge

Philippe DARRIULAT

La place du sentiment national dans les propagandes républicaine et bonapartiste, une étude comparée (1848-1859)

$12 h 00$ 
Conclusion - discussions

$14 h 30$

- Production fonctionnelle de légitimité

Anthony PONCIER

La magistrature contre la République

Patrick LAGOUEYTE

Les magistrats du parquet face au coup d'État

Arnaud-Dominique HOUTE

La gendarmerie au miroir du Deux décembre. Cochet de Savigny et la légitimation du coup d'État

$15 h 40$

Conclusion - discussions

$16 h 40$

Thème 3 : RÉACTIONS EUROPÉENNES

Fabrice BENSIMON

Regards et réactions d'outre-Manche

Antonio DE FRANCESCO

Le coup d'État en Italie

$17 \mathrm{~h} 30$

Conclusion - discussions

Vendredi 30 novembre

$8 h 45$

Thème 4 : RENONCEMENTS, SILENCES ET FIN DES POSSIBLES ?

Présidence et introductions : Alain CORBIN

Conclusions : Corinne SAMINADAYAR-PERRIN

- Renoncements ? - À l'épreuve des romanciers

Patricia BAUDOIN

Balzac et la République : chronique d'une mort attendue

Michèle HECQUET

Sand et les fins de la seconde république : rythmes et scansions du progrès social

Jacques NEEFS

Flaubert sous Napoléon III

$10 \mathrm{~h} 00$

Conclusion - discussions

11 h00

- Continuités et fin des possibles

Laurent CLAVIER \& Jacques ROUGERIE

Entre révolution et coup d'État : production de la République et rapports à l'espace Michèle RIOT-SARCEY

La République, entre social et politique. Le socialisme utopique en question Jacques ROUGERIE

L'association, après et au-delà de Décembre : une continuité progressive

$12 h 10$

Revue d'histoire du XIXe siècle, 22 | 2001 
Conclusion - discussions

$14 h 30$

Alain PLESSIS

Les milieux d'affaires et la mort de la Seconde République

$15 h 00$

Thème 5 : MILITANCES ET RÉSISTANCES : SOCIOLOGIE ET GÉOGRAPHIE

Présidence et introductions : Jean-Luc MAYAUD

Conclusions : Laurent DOUZOU

\section{- Approches locales de la résistance}

Éric DARRIEUX

Territoires ardéchois dans l'insurrection de décembre 1851

Frédéric NÉGREL

Un groupe de résistants anodins : la société secrète montagnarde d'Artignosc

Rémy CAZALS

Dix mille âmes, trente proscrits. Une petite ville industrielle en 1851

$16 h 25$

Christian ESTÈVE

Liberté et droit de chasse : au cœur ou en marge de l'insurrection de décembre 1851 ?

$16 h 45$

Conclusion - discussions

\section{Samedi $1^{\text {er }}$ décembre}

$8 \mathrm{~h} 45$

- Parcours de militances et sociologie d'engagements

Claude-Isabelle BRELOT

Élites déclassées en résistance au coup d'État : une génération perdue?

Laurent LE GALl

Le Finistère après le 2 décembre 1851: révolte impensable, résistance impossible, "militant" improbable?

Michel PIGENET

Devoir de mémoire et fidélité militante. Les noyaux rouges des villages berrichons aux lendemains de la répression de 1851

Louis HINCKER

Veuves, fils et filles des "victimes du 2 décembre". Réflexions sur l'héritage révolutionnaire au $\mathrm{XIX}^{\mathrm{e}}$ siècle à partir du cas parisien

$10 h 35$

Conclusion - discussions

11 h15

Thème 6 : OMBRES ET DESTINS DE 1851

Présidence et introductions : Raymond HUARD

Conclusions : Michel OFFERLÉ

Jean EL GAMMAL 
Le coup d'État du 2 décembre au miroir des cultures politiques en France (fin XIX ${ }^{e}$ début $\mathrm{XX}^{\mathrm{e}}$ siècles)

Maurice AGULHON

Le spectre du Bonapartisme dans l'histoire du XXe siècle français

$14 \mathrm{~h} 00$

Jean GARRIGUES

De Boulanger à de Gaulle : "souviens-toi du deux décembre"

Brigitte GAÏTI

Le fantôme du Deux décembre ou la question de la qualification du 13 mai 1958

$14 h 40$

Conclusion - discussions

$15 h 20$

CONCLUSIONS GÉNÉRALES

- Table ronde, avec

Louis HINCKER, Raymond HUARD, Pierre LÉVÊQUE, Jean-Luc MAYAUD, Michèle RIOTSARCEY

...en dialogue avec la salle 